\title{
Das Zielpublikum „Öffentlichkeit“ als Anknüpfungspunkt für (Medien-) Regulierung
}

\author{
Prof. Dr. Mark D. Cole
}

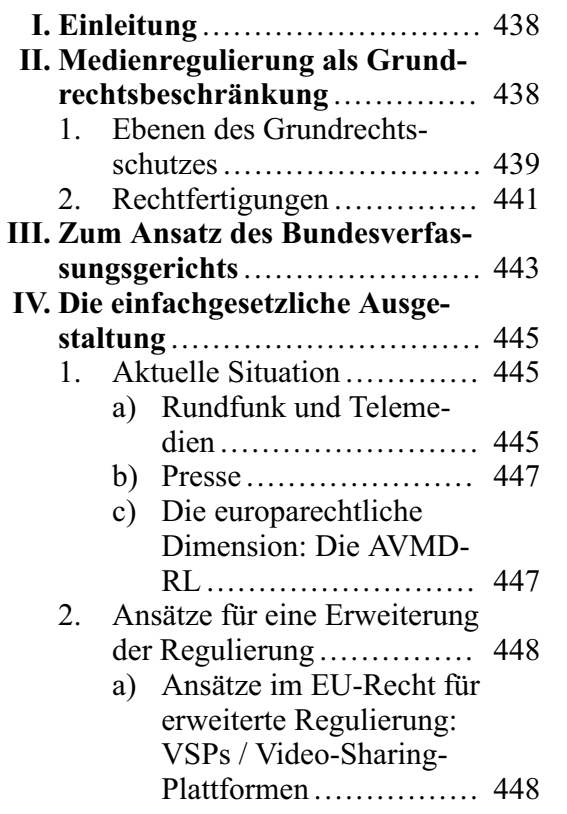

\author{
b) Ansätze im EU-Recht für \\ erweiterte Regulierung: \\ Online-Plattformen I ..... 449 \\ c) Ansätze im EU-Recht für \\ erweiterte Regulierung: \\ Online-Plattformen II .... 450 \\ d) Ansätze im nationalen \\ Recht für erweiterte \\ Regulierung.... \\ e) Rechtsvergleichender \\ Hinweis: US Supreme \\ Court-Urteil
V. ,Öffentlichkeit" und „Impact"6....
2. „Öffentlichkeit“ als Rechts- begriff \\ 1. Ein horizontaler Ansatz...... 454 \\ VI. Fazit \\ 457
}

\begin{abstract}
Der Umgang mit Rechtsproblemen im Zusammenhang mit Inhalten, die über das Internet verbreitet werden, bestätigt, dass Medienrecht als Regulierungsrecht vielfältige Bereiche berührt. Daher lohnt sich bei diesem Rechtsgebiet eine Betrachtung der unterschiedlichen Aspekte in der Zusammenschau. Der Beitrag, der auf einem Vortrag beim „UFITA-Relaunch-Symposium " am 29.6.2018 am Institut für Urheber- und Medienrecht (IUM) in München basiert, gibt einen Anstoß zur Diskussion, ob mittels eines Konzepts der „Öffentlichkeit" ein gemeinsamer Nenner in den Begründungsansätzen von Medienregulierung ge-

* Der Verf. ist Professor für Medien- und Telekommunikationsrecht an der Fakultät für Rechts-, Wirtschafts- und Finanzwissenschaften (FDEF) der Universität Luxemburg und dort auch Fakultätsmitglied am Interdisciplinary Centre Security, Reliability and Trust (SnT); er ist zudem Wissenschaftlicher Direktor am Institut für Europäisches Medienrecht (EMR). Kontakt: m.cole@emrsb.de. Der Beitrag basiert auf dem Vortrag, den der Verfasser beim „UFITA-Relaunch-Symposium“ am 29.6.2018 am Institut für Urheber- und Medienrecht (IUM) in München gehalten hat. Die Vortragsform wurde weitgehend beibehalten und um wenige Fußnoten ergänzt.
\end{abstract}

UFITA 2/2018, DOI: $10.5771 / 2568-9185-2018-2-436$ 
funden werden kann und diese Regulierung somit auf eine einheitliche Basis gestellt werden könnte. Hierzu wird die Frage aufgeworfen, ob und welche übergreifende Rechtfertigung dafür existiert, dass Medien überhaupt reguliert werden. Ziel soll es sein, den Ansatz darauf hin zu überprüfen, ob er die Regulierung neuer Phänomene, die bislang keiner ,,medienspezifischen" Regulierung unterliegen, mit erfassen könnte.

Der Beitrag geht dazu zunächst auf die Ausführungen des Bundesverfassungsgerichts zur Regulierungsnotwendigkeit des Rundfunksektors im nationalen Kontext ein. Dessen Begründung ist maßgeblich von den Merkmalen Breitenwirkung, Aktualität und Suggestivkraft des Rundfunks geprägt. Ein Ansatz, der auch auf online verbreitete Inhalte Anwendung finden könnte, wenn zunächst nur die faktische Lage betrachtet wird. Ähnlich könnte auch der Ansatz zur präventiven Regulierung im Hinblick auf bestimmte Konzentrationsprozesse zur Sicherung der Abbildung einer relevanten Meinungsvielfalt als Überlegung angesichts der ökonomischen Situation im Online-Kontext Anwendung finden.

Anhand der unterschiedlichen einfachgesetzlichen Ausgestaltung bezüglich Presse, Rundfunk und Telemedien auf nationaler wie europäischer Ebene wird der gemeinsame Ausgangspunkt der Inhalteverbreitung und dessen Zielrichtung der Öffentlichkeit herausgearbeitet. Der Beitrag geht dabei auch auf neuere Ansätze zur Erweiterung der bestehenden Medienregulierung im Hinblick auf Online-Angebotsformen ein, die teilweise auf Ebene der EU im Rahmen der AVMD-RL sowie unter dem Stichwort „, Desinformation “ diskutiert oder bereits beschlossen sind. Dabei wird ein Überblick über die relevanten aktuell vorliegenden Vorschläge der Europäischen Kommission gegeben. Auf nationaler Ebene wird eine solche Erweiterung medienbezogener Regulierung anhand der Beispiele Netz-DG und dem geplanten zukünftigen ,, Medienstaatsvertrag “ aufgezeigt.

Nachdem die Vergleichbarkeit mit bisherigen medienrechtlichen Regulierungsansätzen aufgezeigt wurde, geht der Beitrag zur Prüfung auf die mögliche Verwendung auch in diesem Zusammenhang auf die Verwendung des Begriffs „Öffentlichkeit“ in anderen Rechtsgebieten wie dem Strafrecht, Urheberrecht oder Telekommunikationsrecht, aber zum Beispiel auch auf EU-Ebene im Bereich der Terrorismusbekämpfung oder bei der Produktsicherheit ein. Zudem wird untersucht, inwieweit es nicht nur auf die Zielrichtung „Publikum Öffentlichkeit" bei all diesen Ansätzen ankommt, sondern auch dem Merkmal der „Auswirkung “, also inwieweit diese Öffentlichkeit vom jeweiligen Angebot beeinträchtigt werden kann. Im Ergebnis stellt der Beitrag fest, dass für die Zukunft ein horizontaler Ansatz möglich wäre, der den Regulierungsgrund auf die Öffentlichkeitsdimension zurückführt, auch wenn Abgrenzungsfragen der Kriterien „Öffentlichkeit“ und „Auswirkung“ noch offen sind. Auch ist es nicht wahrscheinlich, so das Fazit, dass dies in absehbarer Zeit konkret umgesetzt wird, aber es wäre ein Ansatz, von vornherein regulierungsbedürftige Phänomene zu erfassen, ohne dass es auf eine wie auch immer geartete „sektorspezifische“ Regulierung ankommt, was dann schwierig wird, wenn es keine klar erkennbaren ,Sektoren “ mehr gibt. 


\section{Einleitung}

Medienrecht ist Regulierungsrecht, aber nicht nur. Vielmehr ist Medienrecht eine Querschnittsmaterie, die aus Sicht von Medienanbietern und Mediennutzern vielfältige Rechtsbereiche berührt, die es sich in der Zusammenschau anzusehen lohnt, um der Komplexität des Regelungs“"gegenstands“ Medien gerecht zu werden. ${ }^{1}$ Dies ist keine neue Erkenntnis, zeigt sich aber gerade im Umgang mit Rechtsproblemen im Zusammenhang mit über das Internet oder anders formuliert Online-Plattformen verbreiteten Inhalten.

Im Blick auf das Leitthema dieser Tagung soll dieser Beitrag abschließend eine Art Ausblick darauf geben, ob mittels des/eines Konzepts der „Öffentlichkeit“ Regulierung von Medien oder Phänomenen, die eine Medien vergleichbare rechtliche Einfassung erhalten sollen, auf eine einheitliche Basis gestellt werden kann. Der Beitrag schafft damit sozusagen ein Problem, wo zunächst einmal keines ist, weil jeder Regulierungsakt für sich genommen einer Begründung bedarf und auch vom Gesetzgeber auf nationaler oder europäischer Ebene erhält.

Dennoch soll mit dem Beitrag der Versuch unternommen werden aufzuzeigen, wie diese unterschiedlichen Begründungsansätze möglicherweise schon heute auf einem gemeinsamen Nenner beruhen oder doch auf einen solchen zurückgeführt werden könnten, der dann in Zukunft erlauben würde, in die Öffentlichkeit wirkende Inhaltsangebote oder -anbieter horizontal zu regulieren. Dieser Faktor „Öffentlichkeit“ soll also darauf hin untersucht werden, ob er als Anknüpfungspunkt dafür genommen werden kann, warum wir überhaupt „Medien“ oder allgemeiner gesprochen das Phänomen medialer Verbreitung regulieren. Dies wird an Beispielen auf verfassungs- und einfachgesetzlicher Ebene sowohl aktueller als auch sich entwickelnder Regulierung gezeigt. Auf dieser Basis und im Vergleich zu anderen Rechtsbereichen soll eine erste Einschätzung vorgenommen werden, ob ein solcher auf Öffentlichkeit basierender horizontaler Ansatz möglich und sinnvoll wäre, wobei der Ausblick hin zu etwaigem Forschungsbedarf zeigen wird.

\section{Medienregulierung als Grundrechtsbeschränkung}

Bevor über eine mögliche Veränderung des Begründungsansatzes zur Regulierung von „Medien“ nachgedacht wird, ist der Ausgangspunkt die Frage, warum diese Begründung überhaupt eine Rolle spielt. Da jede Art der Regulierung grundsätzlich eine Grundrechtsbeschränkung darstellt, weil mindestens Entfaltungsmöglichkeiten davon betroffen sind, bedarf diese einer verfassungskonformen Rechtfertigung. Insbesondere wenn es um mediale Kommunikation geht, ist die Regulierung durch staatliche Vorgaben potentiell eine gefährliche Form des Eingriffes, weil sie zunächst einmal geeignet ist, freie Meinungsäußerung

1 Vgl. dazu schon Cole, Handbuch Medienrecht - Recht der elektronischen Massenmedien, 2. Aufl., Frankfurt 2011, Rn. A 1-5. 
und -verbreitung zu verhindern oder zumindest zu behindern. ${ }^{2}$ Deswegen darf es Medienregulierung nicht als Selbstzweck geben, der Staat darf nicht einfach aufgrund einer Neigung zur Regulierung auch den Medienbereich davon erfassen oder - um ein jüngeres Beispiel zu nehmen - Online-Plattformen in eine wie auch immer gestaltete Regulierung einbeziehen. Vielmehr bedarf es einer sorgfältigen Begründung, warum diese Eingriffe notwendig und verhältnismäßig sind. Dafür wiederum gibt es unterschiedliche Begründungsmöglichkeiten, die an der Bedeutung des betroffenen Grundrechts zu messen sind. Auch ist zu berücksichtigen, dass es im Mehrebenensystem des Grundrechtsschutzes auf nationaler und europäischer bzw. internationaler Ebene auch bestimmte Unterschiede gibt.

\section{Ebenen des Grundrechtsschutzes}

Zunächst einmal gibt es die nationale Ebene und hier gilt in Deutschland eine Besonderheit, soweit es um bestimmte Ausprägungen des Rechts auf Meinungsfreiheit geht. Es gehört fast schon zu den Grundlagen des Wissens über Grundrechtsschutz in der Bundesrepublik Deutschland, jedenfalls für ein mit Medienrecht beschäftigtes Publikum, der viel zitierte besondere Auslegungsweg des Bundesverfassungsgerichts bezüglich der Vorschrift des Art. 5 Abs. 1 GG: dieser legt nach der ständigen Rechtsprechung des Gerichts zumindest für den Bereich des Rundfunks eine Ausgestaltungspflicht des Staates fest. ${ }^{3}$ Der Staat ist also - im Rahmen der entsprechenden Kompetenzzuordnung - gehalten, die auf Rundfunk anwendbaren Regeln zu schaffen, indem er eine positive Rundfunkordnung verabschiedet. ${ }^{4}$

Daneben gibt es aber auch die für das Grundrecht zur Meinungsfreiheit und Freiheit der Medien ebenso relevante europäische Ebene. ${ }^{5}$ Dort ist es von großer Bedeutung, wie der Europäische Gerichtshof für Menschenrechte durch seine Rechtsprechung zu Art. 10 EMRK, auch und vielleicht sogar noch mehr in Abgrenzung zum Recht auf Privatheit aus Art. 8 EMRK, indirekt Vorgaben zur Interpretation der Meinungs- und Medienfreiheit macht. ${ }^{6}$ Ebenfalls auf europäischer Ebene, aber innerhalb der Europäischen Union ist der grundrechtliche Einfluss noch größer geworden, seit die Grundrechtecharta der EU Rechts-

2 Vgl. bezogen auf audiovisuelle Medien Weinand, Implementing the EU Audiovisual Media Services Directive, Baden-Baden 2018, S. $49 \mathrm{ff}$.

3 Übersicht zu den relevanten Rundfunkurteilen bei Stettner, in: Hartstein/Ring/Kreile/Dörr/Stettner/ Cole/Wagner (Hrsg.), HK-RStV, 76. AL, Teil B2 Rn. 28 ff. Zur besonderen Rolle des Bundesverfassungsgerichts in dieser Hinsicht anschaulich Dörr, Der Einfluss der Judikatur des Bundesverfassungsgerichts auf das Medienrecht, VerwA 2001, $149 \mathrm{ff}$.

4 Vgl. bereits BVerfGE 12, 205, 261.

5 Überblick dazu Fink/Cole/Keber, Europäisches und Internationales Medienrecht, Heidelberg 2008, Rn. 14 ff.; Oster, European and International Media Law, Cambridge 2017, S. 27 ff.; Dörr, in: HKRStV, Teil B4 Rn. 4 ff.

6 Im Überblick Dörr, in: HK-RStV, Teil B4 Rn. 10. 
wirkung erlangt hat. ${ }^{7}$ Die Charta ist auch deshalb so wichtig, weil dort - anders als in Artikel 10 EMRK oder Art. 5 GG - Absatz 2 eine auf Medien bezogene Besonderheit des Meinungsfreiheitsartikels enthält: „Die Freiheit der Medien und ihre Pluralität werden geachtet “. Unabhängig von der Wirkungsrichtung dieser Vorschrift (wie der Charta insgesamt), die in erster Linie die Organe und Einrichtungen der Europäischen Union bei ihrer Arbeit bindet, unterstreicht die explizite Aufnahme der Medienfreiheit die besondere Bedeutung und Rolle der Medien. ${ }^{8}$ Gerade die Hervorhebung des Werts des Medienpluralismus stellt diesen Wert noch stärker heraus als die nationale Bestimmung, wenngleich das Bundesverfassungsgericht - ebenso wie der Europäische Gerichtshof für Menschenrechte bezogen auf Art. 10 EMRK - Vielfalt der Medien als Gestaltungsziel gesetzgeberischer Maßnahmen (und damit auch entsprechender Beschränkungen der Betätigungsfreiheit z.B. bestimmter Unternehmen) in der Interpretation von Art. 5 GG auch einschließt. ${ }^{9}$

Wenn das Mehrebenensystem betrachtet wird, darf nicht vergessen werden, dass Medienrecht in Deutschland Landesrecht ist, weil die Gesetzgebungskompetenz zur Ausgestaltung von Art. 5 Abs. $1 \mathrm{GG}$ - jedenfalls soweit die inhaltliche Seite betroffen ist - in die Zuständigkeit der Länder fällt. ${ }^{10}$ Bei einem Blick in die Landesmediengesetze finden sich weitere interessante Feststellungen: Beispielsweise im Saarländischen Landesmediengesetz ${ }^{11}$ auf das hier wegen des Sitzes des Instituts für Europäisches Medienrecht in Saarbrücken verwiesen werden soll - ist die besondere Rolle der Medien als Aufgabe, nicht nur für den öffentlich-rechtlichen Rundfunk, sondern für jede Art von Medium und unabhängig von der organisatorischen Gestaltung festgehalten in Art. 4: „Die Medien nehmen bei der umfassenden Teilnahme an der Meinungsbildung eine öffentliche Aufgabe wahr." Nach § 1 Abs. 1 bzw. § 2 Abs. 1 SaarLMG sind damit Presse, Rundfunk, und Telemedien gemeint. Diese Medien betreffen also den Bereich der „Öffentlichkeit“ und haben dafür und daher eine bestimmte Rolle vom Gesetzgeber zugewiesen bekommen.

Ohne dass dies im hiesigen Zusammenhang vertieft werden muss, ist darauf hinzuweisen, dass neben der genannten potentiellen Beeinträchtigung des Grundrechts auf Meinungs- bzw. Medienfreiheit durch eine Regulierung, eine solche auch weitere Grundrechte berührt. Relevant ist dabei vor allem die Berufsausübungsfreiheit oder allgemeiner die Be-

7 Charta der Grundrechte der Europäischen Union, ABl C 83, 30.3.2010, S. 389-403; dazu Dörr, in: HK-RStV, Teil B4 Rn. 11 ff.; Oster/Wagner, E. V. Kommunikation und Medien, in: Dauses/ Ludwigs, Handbuch des EU-Wirtschaftsrechts, München, 31. EL Juli 2012, Rn. 34 ff.

8 Vgl. Calliess/Ruffert/Calliess, EUV/AEUV, 5. Aufl., München 2016, EU-GRCharta Art. 11 Rn. 2 , 16 ff., 30 ff.; BeckOK InfoMedienR/Cornils, 21. Ed. 1.5.2016, EU-GRCharta Art. 11 Rn. 5, 11 ff.; dazu auch Erläuterungen zur Charta der Grundrechte (2007/C 303/02), ABl. C 303/17 v. 14.12.2007, S. 21.

9 Vgl. etwa BVerfGE 31, 314, 326; EGMR, Informationsverein Lentia u.a. gegen Österreich [1993] Beschwerde-Nrn. 13914/88, 15041/89, 15717/89, 15779/89, 17207/90 Rn. 38; dazu Fink/Cole/ Keber, Europäisches und Internationales Medienrecht, Rn. $259 \mathrm{ff}$.

10 Zum Ineinandergreifen der unterschiedlichen Ebenen Cole, in: HK-RStV, B3 Rn. 8 ff.

11 Gesetz Nr. 1490 - Saarländisches Mediengesetz (SMG) Vom 27. Februar 2002, zuletzt geändert durch das Gesetz vom 16. Mai 2018 (Amtsbl. I S. 268). 
rufsfreiheit, die zum Beispiel beschränkt wird, wenn eine nur auf Gewinnerzielungsabsicht ausgerichtete Online-Plattform wegen ihres potentiellen Einflusses zukünftig in einen bestimmten Verantwortlichkeitsstatus gerät, also von einer medienbezogenen Regulierung erfasst würde.

\section{Rechtfertigungen}

Die Beeinträchtigung einer grundrechtlich geschützten Sphäre allein macht staatliche Maßnahmen nicht rechtswidrig. Solange eine Rechtfertigung für die Verkürzung des Grundrechtsschutzes vorliegt und diese verhältnismäßig auch im Einzelfall angewandt wird, bewegt sich der Gesetzgeber bzw. die Exekutive im rechtlich vorgegebenen Rahmen. Auch diese Frage braucht hier nicht vertieft zu werden, denn dass es gute Gründe und rechtfertigungsfähige Möglichkeiten zur Medienregulierung gibt, ist unbestritten. Es gibt Gemeinwohlziele, die geradezu fordern, dass Medien in bestimmtem Umfang reguliert werden. Jugendschutz ist ein oft genanntes Beispiel, nicht nur weil es in der Verfassung als Rechtfertigungsgrund in Art. 5 Abs. 2 GG steht, sondern weil das Schutzinteresse einer gefährdeten Bevölkerungsgruppe staatliche Pflichten auslösen kann. Daher können schon bei herkömmlichen Medien, bei denen eine regelmäßig durch entsprechend ausgebildete Journalisten durchgeführte redaktionelle Inhalteauswahl stattfindet, nicht unlimitiert Inhalte verbreitet werden. Wegen des Gefährdungspotentials für Kinder und Jugendliche wird deshalb nicht darauf abgestellt, dass gegebenenfalls der „Markt der Meinungen“ ungeeignete Inhalte am Ende ablehnt bzw. ausfiltert. Vielmehr wird vorsorglich durch Verbreitungsverbote sichergestellt, dass bestimmte Inhalte schon gar nicht in (frei zugängliche) Medien aufgenommen werden, wie z.B. bestimmte Formen der Pornographie oder weitere in $\S 4 \mathrm{JMStV}$ für Rundfunk und Telemedien genannte Verbotstatbestände. ${ }^{12}$

Ein anderer Rechtfertigungsansatz ist die öffentliche Ordnung als Schutzgut. Dazu gehört etwa, dass bestimmte Äußerungen nicht getätigt werden dürfen, weil sie neben einer möglichen Verletzung individueller Rechtspositionen eine Störung der öffentlichen Ordnung zur Folge haben können. Daher gibt es - zumindest in Europa an vielerlei Stellen verankert $^{13}$ - Verbote für die öffentliche Äußerung von Aussagen, die geeignet sind zum Rassenhass aufzustacheln. Hier unterscheidet sich der Ansatz durchaus erheblich von der Interpretation des ersten Verfassungszusatzes in den USA, der die freie Meinungsäußerung schützt. Demnach könne es die Gesellschaft ertragen, auch mit solchen Meinungsäußerun-

12 Allg. zum jugendmedienschutzrechtlichen Ansatz Cole, Handbuch Medienrecht, Rn. H 2, 4 f.; vgl. auch BVerfGE 83, 130, 139 ff.; ferner zu den Tatbeständen des $§ 4$ Erdemir, in: Bornemann/Erdemir (Hrsg.), Jugendmedienschutz-Staatsvertrag, Baden-Baden 2017, § 4 Rn. 3 ff.

13 So z.B. in Art. 6 AVMD-RL. National können etwa das Verbot der Holocaust-Leugnung in $\S 130$ StGB und vergleichbaren Vorschriften in anderen europäischen Staaten als Beispiele dienen. 
gen konfrontiert zu werden, weil der „Mainstream“ diese am Ende aussortieren wird. ${ }^{14}$ Eine Grenze ist erst erreicht, wenn die Meinungsäußerungen geeignet sind, unmittelbar zu Gewalt gegenüber anderen zu führen. ${ }^{15} \mathrm{Um}$ es zu illustrieren: Während abstrakt gesagt werden kann, dass eine bestimmte Bevölkerungsgruppe schlecht sei, kann man auch in den USA nicht vor dem Wohnhaus einer Person, die zu dieser Bevölkerungsgruppe gehört, eine Hasstirade anstimmen und vor einer gewaltbereiten Gruppe dazu auffordern, Angehörige dieser Bevölkerungsgruppe umzubringen. Dann wird von einem ,imminent threat“, also einer drohenden Gefahr ausgegangen, die die Meinungseinschränkung auch nach dem ansonsten viel weiteren Schutzbereich nach amerikanischer Auffassung rechtfertigt. Eine nach europäischen Maßstäben zum Rassenhass aufrufende allgemeine Äußerung etwa in einem Internetblog ist dagegen in den USA im Regelfall unproblematisch. Genau dieser Ansatz gilt aber in Deutschland und Europa nicht, hier werden vorab Grenzen gesetzt, welche Art von Meinungsäußerungen wegen der Kollision mit anderen (Gemeinwohl-) Interessen zurückzustehen haben.

Die genannten beiden Beispiele sind aber konkrete Rechtfertigungsgründe für Einschränkungen der Meinungsäußerung, auch wenn diese über ein Medium getätigt werden. Der vorliegende Beitrag fragt jedoch grundsätzlicher, welche übergreifende Rechtfertigung dafür existiert, dass Medien reguliert werden. Und wenn es eine solche gibt, welche Angebote bzw. Anbieter alle von dieser Regulierung erfasst sein sollen, also was wir insoweit unter „Medien“ verstehen. Nur um die Frage, ob es einen Ansatz gibt, der auch für andere als die oben genannten Medien Presse, Rundfunk und Telemedien (in der aktuell geltenden Definition) gleichartig verwendet werden kann, soll es gehen. Nicht thematisiert wird dagegen die Folgefrage: Selbst wenn ein horizontaler Ansatz zur Begründung der Regulierung gefunden wird und damit beantwortet werden kann, wer bzw. was reguliert wird, ist dann zu klären, wie reguliert werden soll. Dazu gehören die möglichen Modelle der Regulierung einschließlich Ko- und Selbstregulierungsansätzen, ebenso wie der Umfang von Regulierung. ${ }^{16}$ Diese Anschlussfrage ist interessant und komplex, soll aber deshalb nicht thematisiert werden, weil zunächst davon unbeeinflusst nur geprüft werden soll, ob „Öffentlich-

14 Dazu im Überblick Weaver, Understanding First Amendment, 6th ed., Durham (NC) 2017, S. $11 \mathrm{ff}$. Die konkreten Auswirkungen der unterschiedlichen Maßstäbe zwischen den USA und Europa beleuchtet beispielhaft der Beitrag Cole, The Atlantic or Pacific(a) Divide: On Regulating Broadcast Indecency in the U.S. from a Comparative European Perspective, in: Dörr/Fink/Weaver/Keber (Hrsg.), Brandeis meets Gutenberg, Frankfurt 2012, S. 45 ff.; umfassend Siry, Regulation of Sexualized Speech in Europe and the United States, Baden-Baden 2016, S. $43 \mathrm{ff}, 207 \mathrm{ff}$.

15 Weaver, Understanding First Amendment, S. 21 ff., 34 ff., 141 ff. zsfsd. zur Rechtsprechung des US Supreme Court dazu.

$16 \mathrm{Zu}$ unterschiedlichen möglichen Regulierungsmodellen im Zusammenhang mit Medien Weinand, Implementing the EU Audiovisual Media Services Directive, S. $134 \mathrm{ff}$.; Hans Bredow Institut/ Institut für Europäisches Medienrecht, Final Report, Study on Co-Regulation Measures in the Media Sector, Juni 2006, http://ec.europa.eu/avpolicy/docs/library/studies/coregul/final_rep_en.pdf; Spindler/Thorun, Die Rolle der Ko-Regulierung in der Informationsgesellschaft, MMR-Beilage 2016/1 (Heft 6), 1 ff. 
keit" einen horizontalen Rechtfertigungsansatz begründen kann, der die sektorielle Ausrichtung des bisherigen Medienrechts zumindest dem Grunde nach ablösen könnte.

\section{Zum Ansatz des Bundesverfassungsgerichts}

Für die Frage eines übergreifenden Ansatzes zur Medienregulierung ist zur Erinnerung die Begründung des Bundesverfassungsgerichts heranzuziehen. Dessen Aussagen zur Interpretation von Art. 5 Abs. 1 Satz 2 GG sind zwar alt, aber es lohnt sich immer wieder, sich an diese zu erinnern. Der Begründungsansatz für die Regulierung des Rundfunksektors - und $\mathrm{zu}$ diesem gibt es die am stärksten ausgeprägte Rechtsprechung des Bundesverfassungsgerichts bezüglich Medien und Inhalteverbreitung ${ }^{17}$ - war zunächst klar ein „technischer": Es gab eine Frequenzknappheit für den Übertragungsweg und das musste irgendwie geregelt werden. Wenn jeder das Frequenzspektrum hätte nutzen können, wie er wollte, würde nichts mehr funktionieren, weil kein sauberer Empfang mehr möglich wäre. Schon dieser Ansatz der Organisation einer nur beschränkt verfügbaren Ressource hat genügt, um eine Regulierung zu rechtfertigen. Diese Notwendigkeit hat sich aber in den letzten Jahrzehnten relativiert und dann mit der Digitalisierung jedenfalls weitgehend erledigt. Jedenfalls ist heute das Hauptproblem für die Verbreitung von Medieninhalten nicht mehr ein Zugangsproblem, sondern vielmehr - wie das auch in der Diskussion der vorherigen Beiträge bereits zum Ausdruck gekommen ist - eher die Frage, wie angesichts der zur Verfügung stehenden Informationsbreite und -vielfalt in der Menge an Inhalten sichergestellt werden kann, dass diese auch beim Nutzer ankommen. Daher spielt die Frage des Zugangs auf der Nutzerseite, ob nun technisch oder tatsächlich, heute eine viel wichtigere Rolle. ${ }^{18}$

Trotz der veränderten technischen Lage, hat das Bundesverfassungsgericht aber die Regulierungsnotwendigkeit des Rundfunksektors nie in Frage gestellt und sie im Weiteren mit der bekannten Trias für das Fernsehen begründet, dass das über das Fernsehen übertragene Bewegtbild eine Breitenwirkung, Aktualität und Suggestivkraft habe, die eine gesetzliche Einrahmung des entsprechenden Angebots erfordere. ${ }^{19}$ Wenn nur diese drei Elemente herangezogen und mit den Beispielen in den Vorträgen von Birgit Stark und Felix Stang abgeglichen werden, ${ }^{20}$ so sieht es schon auf den ersten Blick so aus, als könnte dieser Ansatz auch auf online verbreitete Inhalte, die nicht Rundfunk sind aber auch nicht rundfunkähnlich daherkommen, Anwendung finden: Breitenwirkung - wenn man nur die Zahl derer

17 Vgl. zur Bedeutung des BVerfG o. Fn. 3; vgl. ferner eine Zusammenschau bei Cole/Oster, Zur Frage der Beteiligung privater Rundfunkveranstalter in Deutschland an einer staatlich veranlassten Finanzierung, abrufbar unter https://www.prosiebensat1.com/uploads/2017/07/03/P7S1_Rundfunkfi nanzierungBeitrag_Gutachten.pdf, S. $67 \mathrm{ff}$.

18 So jüngst BVerfG, Urteil des Ersten Senats vom 18. Juli 2018 - 1 BvR 1675/16 - Rn. 79.

19 BVerfGE 90, 60, 87.

20 Vgl. dazu Grandjean, Die Bedeutung von Öffentlichkeit und Privatheit im Medien-/Urheberrecht und in der Medienforschung - Tagungsbericht, UFITA 2018, 486 ff.; Stang, Berichterstattung über Social-Media-Inhalte aus urheber- und persönlichkeitsrechtlicher Sicht, UFITA 2018, $375 \mathrm{ff}$. 
nimmt, die die genannten Beispiele konsumieren, wird man nicht bestreiten können, dass eine hohe Nutzerzahl erreicht wird, etwa bei einer Nachricht des bekanntesten „Kurznachrichten-Dienstes“, der in schneller Folge durch Weiterverbreitung an immer mehr Rezipienten ausgesendet wird. Aktualität - oftmals ist die auf dem eben genannten Wege verbreitete Nachricht sozusagen von einer ,überholenden Aktualität“, bei der schneller eine Information weitergeleitet und als solche verkündet wird, als sie sich verifizieren lässt und jedenfalls häufig noch schneller als über Live-Bilder wie im Fernsehen, weil solche nicht überall verfügbar sind, wo Mobiltelefone mindestens eine textliche (oder bildliche) Kommunikation mit der „Außenwelt“ erlauben. Schließlich Suggestivkraft - in Zeiten von „Fake-News“ oder Desinformationskampagnen wird man wohl feststellen können, dass selbst Profijournalisten relativ leicht bzw. häufig auf falsche Agenturmeldungen (weil sie nicht selbst nachgeprüft werden (können)) oder auf nur scheinbare Meldungen etwa im politischen Betrieb „hereinfallen“. Gerade die Suggestivkraft von mit leicht zu manipulierenden Bildern verbundenen Texten, die in starker Verkürzung einen (vermeintlichen) Sachverhalt darstellen, scheint jedenfalls ähnlich wirkungsvoll zu sein wie das (vermeintlich) realitätsabbildende Bewegtbild etwa im Live-Fernsehen. Auch wenn dies eine nicht empirisch abgestützte Beobachtung ist, soll mit dieser kurzen Beleuchtung verdeutlicht werden, dass auch herkömmliche Begründungsansätze für einen Regulierungsbedarf nicht so weit entfernt sind von Phänomenen, die auch bei neuen Inhalteangeboten und -verbreitungsformen auftreten.

Nochmals zur Rechtfertigung der Rundfunkregulierung nach dem Ansatz des Bundesverfassungsgerichts, weil es eine weitere Begründung gibt, die noch genannt werden sollte: Medien sind zwar einerseits Medium für die Meinungsbildung, aber andererseits eben auch Faktor bei eben jener. ${ }^{21}$ Deshalb hat Art. 5 Abs. 1 GG aus der Sicht des Bundesverfassungsgerichts eine objektiv-rechtliche Dimension, zu deren Erfüllung bezogen auf den Rundfunk eine bestimmte Regulierung vorzusehen ist. Weil dieser die erwähnte Aktualität, Breitenwirkung und Suggestivkraft hat, muss er nicht nur im Blick auf den möglichen Einfluss geregelt werden, sondern steht auch in einer besonderen Verpflichtung. Das Konzept einer „dienenden Freiheit" bedeutet, dass der Grundrechtsträger aus der Rundfunkfreiheit nicht so sehr eine subjektiv-rechtliche Position ableiten kann, sondern die objektiv-rechtliche Dimension dazu führt, dass eine das Recht in Anspruch nehmende Person unmittelbar gebunden ist, mit dem Recht auch eine bestimmte Aufgabe zu erfüllen. ${ }^{22}$ Diese Überlegung ist oft kritisiert worden und als eine sehr aufgeladene Interpretation einer grundrechtlichen Bestimmung durch das Bundesverfassungsgericht gesehen worden. ${ }^{23}$ Unbesehen von dieser Kritik hat das Gericht an der Grundidee bis heute festgehalten, wonach der besondere Schutz der Rundfunkfreiheit auch mit einer besonderen Aufgabe einhergeht. ${ }^{24}$ Es wäre si-

21 BVerfGE 12, 205, 260.

22 BVerfGE 57, 295, 320.

23 Vgl. etwa zum dogmatischen Ansatz Hain, Rundfunkfreiheit als „dienende Freiheit“ - ein Relikt?, in: Bitburger Gespräche Jahrbuch 2007/I, S. 22.

24 BVerfG, Urteil des Ersten Senats vom 18. Juli 2018 - 1 BvR 1675/16 - Rn. 77. 
cher interessant zu diskutieren, ob bestimmte Inhaltevermittler neuerer Ausprägung zumindest dem verfassungsrechtlichen Rundfunkbegriff - der weiter ist als die aktuelle einfachgesetzliche Festlegung (dazu unten IV. 1. a)) - unterfallen können, aber hier ging es nur darum aufzuzeigen, dass das Bundesverfassungsgericht wegen der besonderen Auswirkung eines bestimmten Medientyps in der Öffentlichkeit einen Regulierungsbedarf entwickelt hat.

Es gibt weitere Ansätze, die mit hineinspielen bei der Überlegung, warum Rundfunk reguliert werden muss: Der wichtigste ist die Frage der Wettbewerbssituation und insbesondere die Gefahr, die sich daraus ergibt, dass beispielsweise in einem nicht regulierten Markt möglicherweise der Wettbewerb der Meinungen zu sehr von starken Einzelakteuren dominiert werden könnte und es daher keine Angebotsvielfalt mehr gibt. Hier hat das Bundesverfassungsgericht wegen der Unumkehrbarkeit bestimmter Konzentrationsprozesse sehr stark eine präventive Regulierung gefordert. ${ }^{25}$ Insgesamt orientiert sich die Ausgestaltung der Medienordnung daran, dass Vielfalt ein gesellschaftlich wichtiges Ziel ist. Daraus wiederum abgeleitet ist jedenfalls für Deutschland die sehr starke Position des öffentlich-rechtlichen Rundfunks. Allgemeiner formuliert ist die Bedeutung von bestimmten Inhalten, die schlagwortartig als public service- oder public value-Angebote erfasst werden können, und die Qualität bei deren Herstellung ein zu beachtender Faktor, um (relevante) Meinungsvielfalt abzubilden. ${ }^{26}$

Im folgenden Abschnitt wird betrachtet, wie sich diese grundsätzliche Überlegung des Bundesverfassungsgerichts national auf einfachgesetzlicher Ebene niedergeschlagen hat, aber auch, welche Regulierungsansätze auf der EU-Ebene bestehen.

\section{Die einfachgesetzliche Ausgestaltung}

\section{Aktuelle Situation}

\section{a) Rundfunk und Telemedien}

Rundfunk ist einfachgesetzlich definiert und diese Definition hat sich wegen europarechtlicher Einflüsse in den vergangenen Jahrzehnten auch gewandelt. Sie findet sich im relevanten Staatsvertrag, der zwischen allen Ländern vereinbart und ratifiziert worden ist und sich regelmäßig weiterentwickelt: dem „Staatsvertrag für Rundfunk und Telemedien“. ${ }^{27}$ Dieser wird, der offiziellen Abkürzung des Staatsvertrags folgend, üblicherweise „nur“ als Rundfunkstaatsvertrag bezeichnet, was eigentlich schade ist, denn er ist schon lange auch ein

25 BVerfGE 95, 163, 173.

26 Dazu Cole/Oster, Zur Frage der Beteiligung privater Rundfunkveranstalter in Deutschland an einer staatlich veranlassten Finanzierung, S. $17 \mathrm{ff}$.

27 Aktuell in der Fassung des 21. Rundfunkänderungsstaatsvertrags, abgedruckt unter A 1.1 mit der Begründung unter A2.17 bei HK-RStV; Alle Änderungsstaatsverträge und Begründungen finden sich bei HK-RStV, A2. 
Staatsvertrag für Telemedien. ${ }^{28}$ Wobei einschränkend darauf hinzuweisen ist, dass er nicht für alle telemedienbezogenen Fragen gilt, sondern nur für bestimmte Aspekte bzw. bestimmte Telemedien und beachtet werden muss, dass es mit dem Telemediengesetz auch ein Bundesgesetz zu dieser Materie gibt. ${ }^{29}$

Soweit es zunächst um die Definition des Rundfunks geht, findet sich der Öffentlichkeitsbezug in mehreren Aspekten des $\S 2$ Abs. 1 RStV: Er ist ,für die Allgemeinheit" bestimmt und es handelt sich um die „Veranstaltung und Verbreitung von Angeboten“. Es gibt ergänzend die Negativ-Klausel in Absatz 3, die bestimmte Formen von eigentlich der Rundfunkdefinition unterfallenden Informations- und Kommunikationsdiensten vom Anwendungsbereich ausnimmt und auch diese geht von der Frage der Bedeutung in der Öffentlichkeit aus: Kein Rundfunk ist demnach beispielsweise - als eine de minimis-Klausel gegeben, wenn das Angebot nur eine ganz kleine Gruppe betrifft, wenn es weniger als 500 potentielle zeitgleiche Nutzer gibt. Oder wenn es auf eine nur ,persönlichen oder familiären Zwecken" dienende Nutzung abzielt. ${ }^{30}$

Soweit es um Telemedien geht, ist die Definition im Rundfunkstaatsvertrag (auch in $\S 2$ Abs. 1) einerseits sehr weit, andererseits nicht trennscharf, weil eine Negativabgrenzung vorgenommen wurde. Zunächst einmal sind Telemedien „alle elektronischen Informationsund Kommunikationsdienste“ und mit der Bezeichnung ,alle“ ist ein potentiell sehr weiter Anwendungsbereich eröffnet. Telemedien sind solche im Sinne des Staatsvertrags, wenn sie nicht Rundfunk sind - dieser ist im RStV spezieller geregelt - oder keine reine Telekommunikationsdienste - diese werden vom TKG erfasst. ${ }^{31}$ Soweit es sich um ein Telemedium handelt, das also (bezogen auf die Verortung der Regulierung) „weniger“ als Rundfunk und „mehr“ als ein Telekommunikationsdienst ist, wird im materiellen Teil des Rundfunkstaatsvertrages dann aber eine weitere Einschränkung gemacht. Die auf Telemedien anwendbaren Vorschriften sind nämlich bis auf eine Ausnahme beschränkt auf das IV. und die folgenden Kapitel. Die dort zu findenden Vorschriften und insbesondere die spezifischen für die Telemedien im VI. Abschnitt verdeutlichen, dass im Vergleich zum Rundfunk eine wesentlich geringere Regelungsintensität erreicht wird. ${ }^{32}$ Erhöhte Anforderungen gelten nur für eine Unterkategorie von Telemedien, die sogenannten ,journalistisch-redaktionell“ gestalteten, für die etwa journalistische Sorgfaltspflichten gelten ( $\$ 54$ Abs. 2). Ohne hier die Problematik der Bestimmung einer journalistisch-redaktionellen Gestaltung zu behandeln, ${ }^{33}$ liegt hier

28 Die Umbenennung erfolgte mit dem Inkrafttreten des Neunten Rundfunkänderungsstaatsvertrags am 1. März 2007, zu den Hintergründen Cole, in: HK-RStV, B3, Rn. 8, 17 f., 22. Zukünftig soll sich der Name ändern zu Medienstaatsvertrag, dazu Ory, ZUM 2019, Heft 2 und unten IV. 2 d).

29 Zum Anwendungsbereich auf bestimmte Telemedien bezogen vgl. Hartstein, in: HK-RStV, Rn. 5, 8 f., 10 ff.; $\$ 55$ Rn. 8 ff.

30 Umfassend zur Definition Dörr, in: HK-RStV, § 2 Rn 17 ff., 24 ff.

31 Vgl. wiederum Dörr, in: HK-RStV, § 2 Rn $31 \mathrm{ff}$.

32 Hartstein, in: HK-RStV, § 54 Rn 6 f.

33 Vgl. dazu die Beispiele bei Hartstein, in: HK-RStV, § 54 Rn. 15; § 56 Rn. 3 ff. mit Fallgruppen. 
der gesetzgeberische Anlass zur Regelung in der angenommenen vergleichbaren Öffentlichkeitswirkung solcher Telemedien.

\section{b) Presse}

Es stellt sich weitergehend die Frage, wie es mit der einfachgesetzlichen Bestimmung der im Grundgesetz genannten „Presse“ im Blick auf den Öffentlichkeitsbezug ist. Auch die klassische Presse hat einen Regulierungsrahmen, der in entsprechenden Landesgesetzen festgelegt wird, wobei sich dieser vom Rundfunkregulierungsrahmen schon insoweit unterscheidet, als die Pressebetätigung zulassungsfrei ist. ${ }^{34}$ Als Beispiel soll hier $\S 2$ Abs. 2 SaarlLMG genommen werden mit einer Definition, die sich so in den meisten Landesregelungen findet: Es geht bezüglich der Presse um „Druckwerke“. Diese sind ,zur Verbreitung bestimmt"; in anderen Landesmediengesetzen steht sogar ausdrücklich ,zur Verbreitung in der Öffentlichkeit bestimmt". Es wird also wiederum ein Angebot an Inhalten gemacht, das dafür hergestellt worden ist, um in der Öffentlichkeit verbreitet zu werden und dort Wirkung zu entfalten. Auch bezüglich Druckwerken findet sich eine „Ausnahmeklausel“, die bestimmte eigentlich der Definition unterfallende Angebote von den gesetzlichen Bestimmungen ausnimmt. Beispielsweise fallen darunter amtliche Mitteilungen, weil (und jedenfalls soweit) sie keine journalistische Gestaltung haben, oder Mitteilungen die „nur Zwecken des Gewerbes und Verkehrs" (§ 2 Abs. 3 Nr. 2 SaarlLMG) oder im privaten Kontext vervielfältigt bzw. verbreitet werden.

\section{c) Die europarechtliche Dimension: Die AVMD-RL}

Auf der europäischen Ebene finden wir bei der EU „einfachgesetzlich“ (also im Sekundärrecht") die Richtlinie über audiovisuelle Mediendienste (AVMD-RL). Diese ist gerade in überarbeiteter Fassung beschlossen und veröffentlicht worden, nachdem die politische Einigung dazu zwischen Europäischem Parlament und Rat kurz vor dem Sommer erzielt worden war. ${ }^{35}$ Wenngleich die Änderungsrichtlinie (EU) 2018/1808 erst im Dezember 2018 in Kraft tritt und dann in 21 Monaten bis zum 19. September 2020 umzusetzen ist, soll hier schon für die Definitionen auf die neue Fassung Bezug genommen werden.

Ein audiovisueller Mediendienst ist nach Art. 1 Abs. 1 lit. a) ein Dienst, bei dem der Hauptzweck (principal purpose) die Bereitstellung von Sendungen (providing programmes) ist und diese gerichtet sind an die Allgemeinheit (to the general public), also an die allge-

34 Allg. zur Presseregulierung Cornils in: Löffler Presserecht, LPG § 2, Rn. 1 ff.

35 Richtlinie (EU) 2018/1808 des Europäischen Parlaments und des Rates vom 14. November 2018 zur Änderung der Richtlinie 2010/13/EU zur Koordinierung bestimmter Rechts- und Verwaltungsvorschriften der Mitgliedstaaten über die Bereitstellung audiovisueller Mediendienste (Richtlinie über audiovisuelle Mediendienste) im Hinblick auf sich verändernde Marktgegebenheiten, AB1. L 303, 28.11.2018, S. 69-92; dazu Synopse im Vergleich zur aktuell geltenden Fassung: Institut für Europäisches Medienrecht (EMR), AVMD-Synopse 2018, abrufbar unter https://emr-sb.de/ synopsis-avms/. 
meine Öffentlichkeit. Die weiteren in der Definition enthaltenen Kriterien sind im hiesigen Kontext nicht relevant. ${ }^{36}$

Bei den bisher genannten Beispielen zur aktuellen Regelung ist durchgängig erkennbar - und teilweise explizit genannt - der Ausgangspunkt, dass es um Inhalteverbreitungen geht, die darauf ausgelegt sind öffentlich möglichst weit verbreitet zu werden. Im Folgenden werden Ansätze beleuchtet, wie neuere Angebotsformen erfasst werden sollen und ob auch für diese der Öffentlichkeitsbezug gilt.

\section{Ansätze für eine Erweiterung der Regulierung}

Verschiedene Ansätze zur Erweiterung der Regulierung auf neue Online-Angebotsformen, die auf der Ebene der EU diskutiert und teilweise schon beschlossen worden sind, werfen die Frage auf, warum diese Regulierungserweiterung überhaupt in Betracht gezogen wird. Mit dem vorliegenden Beitrag soll genau zur Beantwortung dieser Frage ein Versuch gemacht werden aufzuzeigen, dass es durchaus einen gemeinsamen Faktor zwischen bestehenden und diesen neuen Regulierungsansätzen gibt, der mit dem Merkmal „Öffentlichkeitsgerichtet" beschrieben werden kann.

\section{a) Ansätze im EU-Recht für erweiterte Regulierung: VSPS / Video-Sharing-Plattformen}

In der geänderten AVMD-RL werden zukünftig so genannte VSPs (Video-Sharing-Platforms) miterfasst. In der finalen deutschen Sprachfassung werden diese in Art. 1 Abs. 1 lit. aa) bezeichnet als ,Video-Sharing-Plattform-Dienst“, mithin in einer deutsch-englischen „Melange“, was insofern auffällt, als etwa im Französischen von ,service de plateformes de partage de vidéos" gesprochen wird, also eine taugliche Übersetzung von ,sharing“ existiert bzw. die gängige englischsprachige Abkürzung VSP allein nicht der Grund für diese Textfassung sein kann. Jedenfalls geht es um Plattformen, über die (auch) kurze (von den Plattformnutzern und nicht dem Plattformanbieter hergestellte) Videos (,nutzergenerierte $V_{i d e o s ")}$ ) hochgeladen und verbreitet werden können. Diese Definition ist bereits wesentlich länger als diejenige für audiovisuelle Mediendienste, bezieht sich in den relevanten Kriterien jedoch wiederum auf den „Hauptzweck“ oder die wesentliche Funktion des Angebots, die Bereitstellung des entsprechenden Angebots an die Allgemeinheit sowie einen Einfluss des Plattformanbieters auf die Art der Angebotsdarstellung durch die Organisation des Dienstes. Das bislang gegen die Regulierung von Angeboten wie etwa „YouTube“ vorgebrachte Argument, es handle sich um eine rein technische Plattform, bei der der Anbieter keinen inhaltlichen Einfluss nehme oder nehmen könne, wird mit dieser Definition überholt, indem es nicht mehr darauf ankommt, ob es sich um den Inhalteanbieter selbst han-

36 Umfassend zum Begriff Weinand, Implementing the EU Audiovisual Media Services Directive, S. 292 ff. sowie dies., The revised Audiovisual Media Services Directive 2018 - Has the EU learnt the right lessons from the past?, UFITA 2018, 260, $272 \mathrm{ff}$. 
delt. Das Zurverfügungstellen einer Plattform und die Anordnung der darüber verbreiteten Angebote genügt, um eine in die Öffentlichkeit wirkende Rolle zu bejahen.

Dabei geht die AVMD-RL mit der Reform noch einen Schritt weiter, indem auch die Fernsehähnlichkeit als Grund für die Regulierung zugunsten des Kriteriums der vergleichbaren Wirkung in der Öffentlichkeit abgelöst wird. Schon seit 2007 (mit der letzten Reform der Fernsehrichtlinie hin zu einer AVMD-RL) galt als Regulierungsansatz, dass ein Dienst, der fernsehähnlich daherkommt, zumindest dem Grundsatz nach unter die gleiche Regulierung fallen sollte, weil der Nutzer dies erwarte und es aus seiner Sicht keinen Unterschied machen könne, ob er einen Inhalt direkt konsumiere, wenn er über ein Fernsehprogramm verbreitet wird, oder dieser später auf individuellen Abruf aus einer „Online-Videothek“ verfügbar sei. ${ }^{37}$ Video-on-Demand-Dienste mussten, um audiovisuelle Mediendienste im Sinne der Richtlinie 2007/65/EU (bzw. in der kodifizierten Fassung der Richtlinie 2010/13/EU) zu sein, der Form und dem Inhalt nach mit Fernsehangeboten vergleichbar sein. Dieser Zusatz in der Definition von „Sendung“ in Art. 1 Abs. 1 lit b) fällt mit der neuen Richtlinie (EU) 2018/1808 weg. Auch deshalb können nutzergenerierte Mini-Videoclips wie die viel zitierten „Katzenvideos“ solche Angebote sein, auch weil diese nunmehr ausdrücklich ,unabhängig von ihrer Länge“ als Sendung gelten können. Damit steht im Fokus weniger das „Mediale“ im Sinne des früheren Massenmediums, sondern die Frage, wohin ein Angebot gerichtet ist - geht es aus dem privaten Kontext heraus in die Öffentlichkeit, greift die Regulierungsmotivation.

\section{b) Ansätze im EU-Recht für erweiterte Regulierung: Online-Plattformen I}

Über die Neuregelung der AVMD-RL hinaus spiegelt sich der Ansatz zur Ausdehnung von Regulierung oder der Erwartung bestimmter Verantwortlichkeiten aufseiten der Anbieter in weiteren Rechtsakten bzw. Vorschlägen der EU wider. Auch wenn es keine Verordnung oder Richtlinie und damit „harte Regulierung“ ist, hat die Kommission durch Empfehlungen bzw. Mitteilungen erste Schritte beschlossen, die jedenfalls potentiell - insbesondere wenn diese nicht den erwünschten Effekt erzielen sollten - in eine zukünftige Regulierung in Form von Richtlinien oder Verordnungen führen können.

Im März 2018 hat die Kommission eine Empfehlung ausgesprochen zu ,wirksame[n] Maßnahmen im Umgang mit illegalen Online-Inhalten“. ${ }^{38}$ Dies kann als eine Art „NetzDG light“ bezeichnet werden. Es ist „light“ (leicht) in dem Sinne, dass es kein Gesetz ist, aber es enthält bei allen inhaltlichen Unterschieden ebenfalls eine ganz klare Botschaft an die Plattformen: Diese sollen dafür sorgen, dass illegale Inhalte schneller und effektiver beseitigt werden unter Berücksichtigung widerstreitender Interessen wie insbesondere der Meinungsfreiheit. Mit der Empfehlung (an die Mitgliedstaaten und den Industriesektor) kommt

37 Art. 1 (1) a) i) und Erw.gr. 24 AVMD-RL (2010/13/EU).

38 Empfehlung (EU) 2018/334 der Kommission vom 1. März 2018 für wirksame Maßnahmen im Umgang mit illegalen Online-Inhalten, AB1. 2018 L 63 v. 6.3.2018, S. 50-61. 
zum Ausdruck, dass eine Verbesserung gegenüber der vorher bestehenden Situation erwartet wird und umgekehrt davon ausgegangen werden kann, dass bei einer dauerhaft gleichbleibenden Situation eventuell eine härtere Regulierung folgen wird. ${ }^{39}$

Für den hiesigen Zusammenhang ist nur relevant, wie der Anknüpfungspunkt bestimmt wird. Es geht um Hostingdiensteanbieter, wie sie bereits aus der E-Commerce-Richtlinie ${ }^{40}$ bekannt sind, und die es Nutzern ermöglichen Inhalte zu speichern und dann darüber an Dritte zu verbreiten. Nach Nummer 4 a) der Empfehlung ist es ein „Anbieter von Diensten der Informationsgesellschaft im Sinne des Artikels 14 der Richtlinie 2000/31/EG, der seine Tätigkeit [...] unabhängig vom Ort seiner Niederlassung auf in der Union ansässige Verbraucher ausrichtet". Noch instruktiver ist Erwägungsgrund 13 der Empfehlung, wonach „der zentralen Rolle“ gebührend Rechnung getragen werden soll, „die diese Anbieter beim Ermöglichen einer öffentlichen Debatte sowie der Verbreitung und dem Erhalt" von Inhalten in der Öffentlichkeit „meist“ spielen. Hier wird klar zum Ausdruck gebracht, dass es nicht darum geht eine umfassende Evidenz aus der Medienforschung über die Reichweiten und Wirksamkeit solcher Angebotsformen bereits zu haben, vielmehr genügt das „Bauchgefühl“, dass diese Plattformen bei der Meinungsbildung sehr wichtig sind - jedenfalls meistens. Und das ist auch der Grund sie mit in die Regulierung bzw. an dieser Stelle die erhöhte Verantwortlichkeit einzubeziehen.

Besonders interessant ist dabei, dass es bei den Anbietern nicht mehr darum geht, wo diese ihren Sitz haben, also unter welcher Rechtshoheit sie der Niederlassung wegen stehen, sondern wohin ihr Angebot ausgerichtet ist. Dieses Marktortprinzip ist in vergleichbarer Weise auch in die Datenschutz-Grundverordnung aufgenommen worden ${ }^{41}$ und trägt der Erwartungshaltung Rechnung, dass ein im Rechtsraum der EU-Mitgliedstaaten verfügbares Angebot dem von dieser gesetzten Regelungsrahmen entsprechen soll.

\section{c) Ansätze im EU-Recht für erweiterte Regulierung: Online-Plattformen II}

Es sollen noch drei weitere Beispiele kurz genannt werden, die eine Regulierungserweiterung auf Online-Plattformen im EU-Recht bedeuten bzw. zukünftig bedeuten könnten.

39 Schon die Empfehlung folgt auf eine frühere Selbstverpflichtung verschiedener Unternehmen aus dem Sektor, die für sich genommen allein offensichtlich nicht als ausreichend erachtet wurde, vgl. im Überblick Coche, Privatised enforcement and the right to freedom of expression in a world confronted with terrorism propaganda online, Internet Policy Review, 7(4), DOI: 10.14763/2018.4.1382.

40 Richtlinie 2000/31/EG des Europäischen Parlaments und des Rates vom 8. Juni 2000 über bestimmte rechtliche Aspekte der Dienste der Informationsgesellschaft, insbesondere des elektronischen Geschäftsverkehrs, im Binnenmarkt („Richtlinie über den elektronischen Geschäftsverkehr"), ABl. OJ L 178, 17.7.2000, S. 1-16.

41 Art. 3 (2) und Erw.gr. (23) Verordnung (EU) 2016/679. 
Zunächst gibt es eine weitere Mitteilung vom April dieses Jahres ${ }^{42}$ die sich auf sogenannte „Fake-News“ bezieht oder, wie es in der Mitteilung nun heißt, „Desinformation“ im Internet. Diese Mitteilung ist ein „soft law“-Ansatz, der aber auch mit der Aussage an die Plattformen gerichtet ist, dass diese wegen ihres Beitrags zur Meinungsbildung in der Öffentlichkeit eine Rolle spielen müssen bei der Bekämpfung von Desinformation. Dazu gehört die Erwartungshaltung der Entwicklung eines Verhaltenskodexes, der innerhalb kürzester Zeit vorzulegen war. ${ }^{43}$ Dieser unter anderem die Praxis von Facebook, Twitter und Google widerspiegelnde Kodex ist im September 2018 öffentlich gemacht worden und enthält einen Annex, der konkret unternehmensbezogen die Aktivitäten dokumentiert. ${ }^{44}$ Ferner geht die Empfehlung weiter und kündigt eine Überprüfung der Maßnahmen bereits am Jahresende an, um im Blick auf die Wahlen zum Europäischen Parlament im kommenden Jahr $\mathrm{zu}$ entscheiden, ob weitere Maßnahmen nötig sind. Entscheidend ist, dass den Plattformen als Informationsverbreitern eine wichtige Wirkungsmacht in der öffentlichen Debatte und damit die Gefahr einer Verzerrung bei der Meinungsbildung bei Verbreitung von „falschen Informationen" zuerkannt wird.

Auch wenn die bis hierhin genannten Beispiele außerhalb der AVMD-RL noch nicht Rechtsakte mit Bindungswirkung vergleichbar einer Richtlinie sind, sollte nicht verkannt werden, dass insgesamt die Rolle der Plattformen auch gesetzgeberisch anders betrachtet wird als noch vor einigen Jahren. ${ }^{45}$ In der EU zeigt sich das auch daran, dass eine der Maßnahmen zur Förderung der Digitale Binnenmarkt-Strategie der Kommission bereits zu einem Vorschlag für einen Regelungsakt in Form einer Verordnung bezüglich der Plattformen geführt hat: Die Verordnung zur Förderung von Fairness und Transparenz für gewerbliche Nutzer von Online-Vermittlungsdiensten ${ }^{46}$ soll im Business-to-Business Verhältnis sicherstellen, dass keine unzulässige Diskriminierung stattfindet, also etwa in der Abbildung von Suchergebnissen. Der im April 2018 vorgelegte Vorschlag ist noch im Gesetzgebungsverfahren, wird aber ein weiterer Baustein sein, Plattformen wegen ihrer Öffentlichkeits-

42 Mitteilung der Kommission an das Europäische Parlament, den Rat, den Europäischen Wirtschafts- und Sozialausschuss und den Ausschuss der Regionen, Bekämpfung von Desinformation im Internet: ein europäisches Konzept, COM/2018/236 final, abrufbar unter https://eur-lex.europa. eu/legal-content/DE/TXT/?uri=CELEX\%3A52018DC0236.

43 Vgl. Überblick https://ec.europa.eu/digital-single-market/en/news/code-practice-disinformation. Vgl. ferner zur Thematik insgesamt Ukrow/Etteldorf, „Fake News“ als Rechtsproblem, EMR Script Bd. 5, Saarbrücken 2018.

44 Kodex abrufbar unter https://ec.europa.eu/newsroom/dae/document.cfm?doc_id=54454 und Annex https://ec.europa.eu/newsroom/dae/document.cfm?doc_id=54455.

45 Einen detaillierten Überblick über sektorale Regulierungsansätze geben Quintel/Ullrich, in: Ojanen/Petkova, Fundamental Rights Protection Online: the Future Regulation of Intermediaries, Edward Elgar, 2019 (im Erscheinen; Vorab-Version unter: https://papers.ssrn.com/sol3/papers.cfm?ab stract_id=3298719) und Ullrich, Standards for Duty of Care? Debating Intermediary Liability from a Sectoral Perspective, JIPITEC 8(2) 2017, $111 \mathrm{ff}$.

46 Vorschlag für eine Verordnung des Europäischen Parlaments und des Rates zur Förderung von Fairness und Transparenz für gewerbliche Nutzer von Online-Vermittlungsdiensten, COM/ 2018/238 final. 
wirksamkeit zu adressieren. In diesem Zusammenhang wird es zwar um einen unverzerrten Wettbewerb und nicht die Frage des Einflusses auf die öffentliche Meinungsbildung gehen, aber auch hier ist der Anlass die wichtige Funktion der Online-Vermittlungsdienste und Suchmaschinen auf dem Weg zwischen Anbieter eines Inhalts und Rezeption in der Öffentlichkeit, in dem Fall also Kunden in der EU.

Schließlich kann noch auf den Vorschlag vom September 2018 verwiesen werden für eine Verordnung zur Verhinderung der Verbreitung terroristischer Online-Inhalte. ${ }^{47}$ Es geht wiederum im Vorschlag nach Art. 1 (2) um Hostingdiensteanbieter, die nach Art. 2 Abs. 1 dritten Personen und damit der Öffentlichkeit den von anderen hochgeladenen Inhalt zugänglich machen, wobei als ein Fall dafür (nach Art. 2 Abs. 3 lit.b)) die erhebliche Zahl von Nutzern in einem oder mehreren EU-Mitgliedstaaten genannt wird. Die Bekämpfung der Verbreitung von terroristischen Inhalten soll unter Einbeziehung der Plattformen stattfinden, weil diese durch ihren Organisationsanteil einerseits effektive Maßnahmen treffen können und andererseits wegen ihrer Breitenwirkung das Verfügbarsein eines entsprechenden Inhalts auf der Plattform als gefährlich eingeschätzt wird. ${ }^{48}$

\section{d) Ansätze im nationalen Recht für erweiterte Regulierung}

Nur der Vollständigkeit halber soll noch darauf hingewiesen werden, dass die Erweiterung medienbezogener Regulierung auch auf nationaler Ebene stattfindet. Dazu sollen zwei Beispiele nur kurz genannt werden.

Das bereits intensiv diskutierte Gesetz zur Verbesserung der Rechtsdurchsetzung in sozialen Netzwerken, das NetzDG, ${ }^{49}$ kommt zuerst in den Sinn, wenn an erweiterte Verantwortlichkeiten von (jedenfalls bestimmten) Plattformanbietern gedacht wird. Inhaltlich soll dies hier nicht näher dargestellt, aber wiederum der Anwendungsbereich betrachtet werden: das Gesetz gilt nach $\S 1$ Abs. 1 für ganz bestimmte Telemediendiensteanbieter, deren mit Gewinnerzielungsabsicht angebotene Plattformen Nutzern das Teilen von Inhalten mit anderen Nutzern ermöglichen oder diese „,der Öffentlichkeit zugänglich“ zu machen. Auch

47 Vorschlag für eine Verordnung des Europäisches Parlaments und des Rates zur Verhinderung der Verbreitung terroristischer Online-Inhalte - Ein Beitrag der Europäischen Kommission zur Tagung der Staats- und Regierungschefs vom 19.-20. September 2018 in Salzburg, COM/2018/640 final.

48 Der Vorschlag ist vor allem deshalb hervorhebenswert, da er erstmals Plattformen spezifische prozedurale Vorgaben zur vorbeugenden Bekämpfung von terroristischen Inhalten macht, vgl. COM/ 2018/640 final, Erwägungsgründe 16-18 bzw. Artikel 6.

49 Gesetz zur Verbesserung der Rechtsdurchsetzung in sozialen Netzwerken (Netzwerkdurchsetzungsgesetz-NetzDG), BGB1. I, Nr. 61, Seite 3352; dazu Hofmann, Die Konturierung der „öffentlichen Wiedergabe" und des Öffentlichkeitsbegriffs durch den EuGH, UFITA 2018, $334 \mathrm{ff}$.; vgl. ferner die Beiträge in Eifert/Gostomzyk (Hrsg.), Netzwerkrecht - Die Zukunft des NetzDG und seine Folgen für die Netzwerkkommunikation, Baden-Baden 2018 sowie Müller-Franken, Netzwerkdurchsetzungsgesetz: Selbstbehauptung des Rechts oder erster Schritt in die selbstregulierte Vorzensur? - Verfassungsrechtliche Fragen, AfP 2018, 1 ff.; Peifer, Netzwerkdurchsetzungsgesetz: Selbstbehauptung des Rechts oder erster Schritt in die selbstregulierte Vorzensur? - Zivilrechtliche Aspekte, AfP $201814 \mathrm{ff}$. 
hier ist unterstrichen, dass es nicht so sehr um den Einzelaustausch von Inhalten zwischen Nutzern geht, sondern über diese Plattformen öffentliche „Debatten“ geführt werden, weil Informationen breit geteilt werden. Es ist aber darauf hinzuweisen, dass von den neu geschaffenen Pflichten des Gesetzes nur Plattformen erfasst werden, die als soziale Netzwerke mehr als zwei Millionen registrierte Nutzer in Deutschland haben, was den Anwendungsbereich einschränkt.

Die Rolle der Plattformen wird zukünftig auch in einem Rundfunkänderungsstaatsvertrag neu adressiert werden. Die Plattformregulierung (verstanden im bisherigen nationalen Sinne des Rundfunkstaatsvertrags, wie sie im Zehnten Abschnitt niedergelegt ist) soll signifikant ausgeweitet werden, wobei nicht nur herkömmliche Plattformen, wie Kabelnetzbetreiber, sondern z.B. auch Hardware-Hersteller von Endgeräten erfasst werden können. Dies alles soll in einem „Medienstaatsvertrag“ aufgegriffen werden - in den ersten Diskussionen wurde dieser noch als „Modernisierungsstaatsvertrag“ bezeichnet -, für den ein erster Entwurf im Sommer 2018 von den Ländern vorgelegt wurde und seither in einem Konsultationsprozess ist. ${ }^{50}$ Die Motivation der Ausdehnung der Regelung im gleichen Staatsvertrag, der Rundfunk und Telemedien regelt, gründet auf der Wirkungsweise dieser Plattformen bei der Distribution und dem Empfang von (medialen) Inhalten, die bei der öffentlichen Meinungsbildung entscheidend sind.

\section{e) Rechtsvergleichender Hinweis: US Supreme Court-Urteil}

Ohne dass dies hier systematisch erfolgt, können auch in unserem Zusammenhang rechtsvergleichende Einblicke von Interesse sein. Aus Gründen des Umfangs soll arbiträr ein Beispiel aus den USA genommen werden, das bestätigen kann, wie „Öffentlichkeit“ als Faktor bzw. Kriterium bei Regelung von Plattformen genutzt werden kann.

Der US Supreme Court hat letztes Jahr ein interessantes Urteil in Packingham v North Carolina gefällt. ${ }^{51}$ Es gab ein Gesetz in North Carolina, welches verurteilten Sexualstraftätern den Gebrauch von Online-Diensten untersagte, die auch von Minderjährigen genutzt werden. Der Schutzzweck des Gesetzes ist ganz klar, was der Supreme Court anerkennt, indem er die Motivation des Gesetzgebers als nachvollziehbar einordnet. Dennoch hat er das Gesetz gekippt, weil es viel zu breit (,overbreadth') angelegt ist. Die Schwere des Eingriffs in die Meinungsfreiheit dieser verurteilten Straftäter begründet der Supreme Court mit der Bedeutung von sozialen Netzwerken: ,for many [these] are the principal sources for knowing current events,..., speaking and listening in the modern public square,... [they] provide perhaps the most powerful mechanisms available to a private citizen to make his or her voice heard...". Die sozialen Netzwerke haben aus Sicht des Supreme Court also eine so wichtige Bedeutung bei der öffentlichen Verkündung einer Meinungsäußerung erlangt (,ef-

50 Vgl. dazu www.rundfunkkommission.rlp.de sowie Beiträge aus der Tagung des IUM am 9. November 2018, in ZUM 2019, Heft 2.

51137 S. Ct. 1730 (2017). Dazu Weaver, Friedland u.a., Constitutional Law: Cases Materials and Problems, Fourth Edition, 2018 Supplement, New York, S. 190 ff. 
fektivster Weg für Individuen sich bemerkbar zu machen“), dass ein so breit gefasstes Gesetz nicht möglich ist. Einschränkungen seien möglich, aber der Gesetzgeber dürfe nicht alle sozialen Netzwerke, bei denen auch Minderjährige sind, davon erfassen. Der Supreme Court ist in vielem sehr konservativ. Die sozialen Netzwerke als diejenigen zu bezeichnen, die heute Öffentlichkeit herstellen, ist daher eine bemerkenswerte Feststellung.

\section{V. „Öffentlichkeit"“ und „Impact“"}

\section{Ein horizontaler Ansatz}

Der bisherige Überblick über Regulierungsansätze diente dazu zu illustrieren, dass bereits jetzt eine Vergleichbarkeit darin liegt, dass die erfassten Anbieter bzw. Angebote eine Rolle in der Öffentlichkeit haben bzw. in diese hineinwirken. Es lässt sich ergänzen: und dort einen „Impact“ haben, also eine nennenswerte Auswirkung. Genau dies könnte Grundlage für einen horizontalen Begründungsansatz für (,Medien“-)Regulierung sein.

Dieser horizontale Ansatz sollte ermöglichen, dass es nicht (mehr) auf die verwendete Technik ankommt. Es soll nicht auf die typische Erscheinungsform ankommen, also etwa ob es rundfunkähnlich ist oder von der Regulierungsseite her ob es eine Lizenzanforderung gibt. Auch nicht sollte entscheidend sein, von wem das Angebot kommt, also z.B. ob es professionell ausgebildete Journalisten sind, die den Inhalt produziert haben. Stattdessen würde darauf abgestellt werden, dass die Zielrichtung eines Angebots, der Adressat einer Inhalteverbreitung die Öffentlichkeit ist. Und das sollte dann zunächst möglichst breit ausgelegt werden. Dadurch würden mehr Inhalte darunterfallen als bislang. Eine Einschränkung könnte ergänzt werden, indem nur diejenigen öffentlich gemachten Inhalte zählen, die eine nennenswerte Auswirkung haben. Dabei wäre aber noch zu klären, ob sowohl hinsichtlich der Rezeptionsbasis als auch der Auswirkung die subjektive Intention des Inhalteerstellers bzw. -anbieters entscheidend ist, oder objektive Kriterien. Um zu verhindern, dass (gewissermaßen vergleichbar mit der bislang oft argumentierten „Plattformlogik“) insbesondere die Verbreiter sich darauf zurückziehen, eine Beteiligung am öffentlichen Meinungsbildungsdiskurs sei selbst gar nicht gewollt, muss es um die Entwicklung objektiver Kriterien gehen, die die beiden Merkmale handhabbar machen. ${ }^{52}$

\section{2. „Öffentlichkeit“" als Rechtsbegriff}

Dass ein solcher Ansatz durchaus auf vorhandene Überlegungen aufbauen kann, liegt auch daran, dass der Begriff der Öffentlichkeit nicht nur im Medienrecht eine Rolle spielt. Ohne systematische Auswertung sollen hier nur einige Beispiele genannt werden, wo Öffentlichkeit eine (gesetzliche) Rolle spielt, was dann vielleicht auch nutzbar gemacht werden könnte, wenn das „Öffentlichkeitsmerkmal“ für unseren Bereich zugeschnitten wird.

52 Vgl. dazu etwa Helberger, in: Moore/Tambini (Hrsg.), Digital Dominance, New York 2018, S. $153 \mathrm{ff}$. 
Das Urheberrecht ist naheliegend und war umfänglich Gegenstand der Tagung, weil dort die Verbreitung an die Öffentlichkeit von grundlegender Bedeutung ist. ${ }^{53}$ Aber man kann auch in das Strafrecht blicken, etwa in den Tatbestand der Erregung öffentlichen Ärgernisses nach § 183a StGB. Bestimmte sexuelle Handlungen kann man zu Hause vornehmen, so viel und in der Art und Weise wie gewünscht. Strafrechtlich relevant werden kann es aber, wenn diese Handlung mit Relevanz für die Öffentlichkeit vorgenommen wird. Das bedeutet, es muss nicht notwendigerweise im öffentlichen Raum stattfinden. Das kann auch im privaten Bereich erfolgen, aber durch die „öffentliche Verbreitung“ eine ebensolche Wirkung haben, beispielsweise wenn durch ein Fenster die Handlung in der privaten Wohnung bewusst so sichtbar gemacht wird, dass z.B. Mitarbeiter in unmittelbar gegenüber liegenden Büros gleichsam wie im Fernsehen zuschauen können. ${ }^{54}$ Umgekehrt kann auch eine Handlung im öffentlichen Raum unproblematisch sein, wenn sie in einem abgeschiedenen Bereich stattfindet. Es geht also darum, welche (potentiellen) Auswirkungen die Aktivität haben kann, ob also die Öffentlichkeit davon gestört wird oder nicht.

Das Telekommunikationsrecht bezieht sich auf Netze, die öffentlich zugänglich sind, wie es in $\S 3$ Nr. 17a TKG heißt. Die Begrifflichkeit greift die Vorgaben aus dem EU-Recht auf, früher wurde im TKG der Begriff „Telekommunikationsdienste für die Öffentlichkeit“ mit gleicher Bedeutung verwendet. Ein Netz wird „öffentlich“, wenn es nicht nur einem begrenzten Nutzerkreis zur Verfügung steht. ${ }^{55}$

Auch im Straßenverkehrsrecht knüpfen viele der Regelungen daran an, dass sich der Sachverhalt auf einer öffentlich zugänglichen Straße abspielt. So spielt das Verbot des Fahrens in betrunkenem Zustand erst dann eine Rolle, wenn das Kraftfahrzeug im öffentlichen Raum bewegt wird. Darauf bezogen sind die Gerichte in der Auslegung aber eher streng, denn eine vermeintlich private Straße ist keine solche, wenn man diese grundsätzlich benutzen kann oder wenn der Nutzerkreis nicht ein kleiner abgeschlossener, durch persönliche Beziehungen verbundener Kreis ist. ${ }^{56}$ Sobald eine Gefahr „,in der Öffentlichkeit“ gesetzt wird, sollen ordnungswidrigkeiten- oder strafrechtliche Sanktionen greifen können.

Im EU-Recht findet sich der Anknüpfungspunkt „Öffentlichkeit“ ebenfalls außerhalb der oben genannten medienbezogenen Beispiele. Noch vor dem Vorschlag für eine Verordnung zur Bekämpfung terroristischer Inhalte online (dazu oben IV. 2. c)), trat 2017 die

53 Dazu die Beiträge von Hofmann, Die Konturierung der „öffentlichen Wiedergabe“ und des Öffentlichkeitsbegriffs durch den EuGH, UFITA 2018, 334 ff.; Jütte, Ein horizontales Konzept der Öffentlichkeit - Facetten aus dem europäischen Urheberrecht, UFITA 2018, 354 ff.; Dreier, in: Dreier/Schulze, Urheberrechtsgesetz, 5. Auflage, München 2015,§ 15 Allgemeines, Rn. 37 ff.

54 BGHSt 11, 282; Eisele, in: Schönke/Schröder, Strafgesetzbuch, 29. Aufl., München 2014, § 183a StGB Rn. 4.

55 Schütz, Beck'scher TKG-Kommentar, 4. Aufl., München 2013, § 3, Rn. 59.

56 OLG Düsseldorf, NJW 1956, 1651. 
Richtlinie zur Terrorismusbekämpfung in Kraft. ${ }^{57}$ Diese ist weit formuliert, indem sie in Art. 5 öffentliches Verbreiten oder sonstige öffentliche Zugänglichmachung von Äußerungen, die zu Terrorismus führen können als von den Mitgliedstaaten strafrechtlich streng zu ahndende Tatbestände vorsieht. Anlass ist hier wiederum, dass das öffentliche Zugänglichmachen solcher Äußerungen als sehr gefährlich gewertet wird. Artikel 21 sieht daher vor, dass die Mitgliedstaaten unterschiedliche Maßnahmen zur Beseitigung dieser Inhalte vorsehen müssen, die gegebenenfalls auch über den Hostinganbieter gehen müssen. ${ }^{58}$ Interessant ist Erwägungsgrund 10 der Richtlinie, der die öffentliche Aufforderung zur Begehung der Straftat näher beschreibt. Eine Gefahr, dass dies zu einer entsprechenden Handlung führt, soll bejaht werden, wenn die konkreten Umstände des Falles dies annehmen lassen. Dabei soll nicht nur auf den Urheber der Nachricht, sondern auch auf den Empfänger geachtet werden. Wenn der Urheber oder Verbreitende die Zielrichtung hat, dass möglichst viele den Inhalt zur Kenntnis nehmen und danach handeln, dann ist das Öffentlichkeitsmerkmal gegeben, insbesondere wenn das im Empfängerkreis zu einem vom Urheber gewünschten Ergebnis führen kann.

Als letztes Beispiel soll eine Regelung zur Produktsicherheit herangezogen werden. Nach einem Beschluss, der den Rechtsrahmen für die Vermarktung von Produkten festlegt ${ }^{59}$ kommt es für die Frage der Verantwortlichkeit darauf an, wer zuerst das Produkt auf den Markt bringt - formuliert als „Abgabe“ bzw. „erstmalige Bereitstellung“ (Anh. I Art. R1). Dort ist zwar der Begriff „Öffentlichkeit“ nicht verwendet, aber es geht genau darum. Wer ein Produkt auf den Markt und damit in die Öffentlichkeit bringt, schafft die potentielle Gefahr und ist daher für das Produkt verantwortlich. Der Schutz des Konsumenten als öffentliches Interesse bedingt die Regelung, wenn mit dem Konsumenten als Teil der Öffentlichkeit eine Beziehung entsteht. Dabei ist es interessant festzustellen, dass die Verantwortlichkeiten von E-Commerce Plattformen beim Inverkehrbringen und Vermarkten von Produkten ebenfalls diskutiert wird. ${ }^{60}$

57 Richtlinie (EU) 2017/541 des Europäischen Parlaments und des Rates vom 15. März 2017 zur Terrorismusbekämpfung und zur Ersetzung des Rahmenbeschlusses 2002/475/JI des Rates und zur Änderung des Beschlusses 2005/671/JI des Rates, AB1. L 88 vom 31.3.2017, S. 6-21.

58 Vgl. ergänzend Empfehlung (EU) 2018/334 (o. Fn. 38), Nr. 35 zur Entfernung terroristischer Inhalte in der Regel bis eine Stunde nach Meldung.

59 Beschluss Nr. 768/2008/EG des Europäischen Parlaments und des Rates vom 9. Juli 2008 über einen gemeinsamen Rechtsrahmen für die Vermarktung von Produkten und zur Aufhebung des Beschlusses 93/465/EWG des Rates (Text von Bedeutung für den EWR), AB1. L 218 vom 13.8.2008, S. $82-128$.

60 So im Vorschlag für eine Verordnung des Europäischen Parlaments und des Rates zur Festlegung von Bestimmungen und Verfahren für die Konformität mit und die Durchsetzung von Harmonisierungsrechtsvorschriften der Union für Produkte [und Änderungen an Richtlinien], COM/2017/795 endg., Erwägungsgründe 11-13 i.V.m. Artikel 3 (1), (12) lit. h; vgl. ferner Leitlinien zur Umsetzung/Anwendung der Richtlinie 2005/29/EG über unlautere Geschäftspraktiken, Begleitunterlage zur Mitteilung der Kommission „Ein umfassendes Konzept zur Förderung des grenzüberschreitenden elektronischen Handels für die Bürger und Unternehmen Europas“, SWD/2016/163 endg., Ab- 


\section{Fazit}

Zusammenfassend ist den genannten Ansätzen die Zielrichtung gemein, die Öffentlichkeit zu schützen, wenn etwas in die Öffentlichkeit ,gelangt“. Das wäre daher ein Ansatz, der auch umfassend bei der Medienregulierung (im weit verstandenen Sinne) genutzt werden könnte. Es geht hier nicht darum, eine Ablösung bisheriger Kategorien oder Kriterien als zwingend einzuordnen. Im Gegenteil haben die unterschiedlichen Regelungswerke für sich genommen in der Vergangenheit funktioniert. Die Idee der Suche nach einem ,gemeinsamen Nenner" - hier der Wirkung in der Öffentlichkeit - ist vielmehr aufzuzeigen, dass für die Zukunft ein horizontaler Ansatz möglich wäre, der den Regulierungsgrund auf die Öffentlichkeitsdimension zurückführt. Soweit der Regelungsbedarf für „klassische“ Medien erkannt wurde, müsste demnach die Einwirkung auf die Öffentlichkeit auch für jede andere Form der Inhalts- und Meinungsverbreitung zumindest als Grund für eine wie auch immer geartete Regulierung gelten.

Wer die Gegenfrage stellen möchte, wie wahrscheinlich es ist, dass ein solcher Ansatz in den kommenden Jahren umgesetzt wird, bekommt die Antwort, dass es sicher nicht kurzfristig angegangen wird. Die medienpolitische Diskussion wird trotz aller Konvergenz oft noch - und im Sinne der Erreichung politischer Kompromisse nachvollziehbar - an den Linien bestehender Gesetzgebung, die sektoriell ausgerichtet war, geführt. ${ }^{61}$ Aber der Ausblick am Ende einer Tagung wie dieser, darf auch etwas weiter in Richtung Horizont erfolgen. Und dabei darf dann eines wiederum nicht unbeachtet bleiben: Die Ausgestaltung einer Regulierung auf der Basis von Öffentlichkeit und Wirkung müsste dann eine sehr genaue Grundrechtsabwägung vornehmen. Hinsichtlich der Anforderungen und Pflichten wäre dann zwischen unterschiedlichen Angeboten zu unterscheiden, es wäre sicherlich so, dass auch weiterhin unterschiedliche Angebotsformen andere Regelungserfordernisse erfüllen müssten. So wäre etwa die Übertragung einer Lizenznotwendigkeit auf jeden Anbieter, der in der Öffentlichkeit Informationen verbreitet mit dem Argument, auch der Rundfunkveranstalter habe ja eine Lizenz gebraucht, nicht möglich. In der Grundrechtsabwägung müssten auch die Regulierungsdichte und -form wie z.B. Möglichkeiten regulierter Selbstregulierung miteinbezogen werden.

Sind denn die beiden genannten Kriterien „(Teil-) Öffentlichkeit“ und „(Aus-) Wirkung“ überhaupt tauglich? Für beide können die regelnden Juristen von Erkenntnissen der Medienforschung profitieren. Auf den ersten Blick ist das Kriterium der Öffentlichkeit relativ klar fassbar und kann auf bestehenden Ansätzen aufbauen. Es müsste zwar festgelegt werden, wo der rein private Bereich endet, wo es in einen Mischbereich der „Semi-Öffentlichkeit“"geht, aber solche Abgrenzungsfragen sind nicht ungewöhnlich und machen ein

schnitt 5.2. Allg. dazu Ullrich, New Approach Meets New Economy - Enforcing EU Product Safety in E-Commerce, eingereicht zur Veröffentlichung 2019, Manuskript, S. 12-14.

61 Vgl. zuletzt bei der Diskussion um den 22. RÄndStV zur Definition des Telemedienauftrags öffentlich-rechtlicher Rundfunkanstalten und wie dort letztlich die bisherigen Kategorien Rundfunk, Telemedien und Presse bzw. Presseähnlichkeit bei der Grenzziehung beibehalten wurden. 
solches Kriterium nicht unscharf. Schwieriger könnte es mit dem Kriterium der Auswirkung werden, weshalb hier in Zukunft weiter geforscht werden muss. Es gibt im OnlineKontext noch nicht ausreichend objektivierte Messfaktoren für die genaue Festlegung der Auswirkung eines Inhalts. Andererseits verlangt Medienregulierung (bzw. die Begründung für eine Regulierung) nicht, dass eine Auswirkung genau bewiesen werden kann. Medienregulierung hat immer auch auf prognostischen Aussichten aufgebaut. Im Jugendmedienschutzrecht etwa ist weitgehend unbestritten, dass es zu riskant wäre auch ohne vollständige Evidenz Inhaltekonsum für Kinder und Jugendliche unreguliert zu lassen, weil der mögliche Schaden zu groß wäre. ${ }^{62}$ Als Konsequenz trifft der Gesetzgeber dann auch die Entscheidung, bestimmte Inhalte von einer Verbreitung fern zu halten. Ein „Langzeittest“ an Personen der Zielgruppe bezüglich der Auswirkung von als ungeeignet erkannten Inhalten auf einen Minderjährigen verbietet sich von selbst. Damit könnte auch ohne detaillierte Erkenntnis zur Medienwirkung bzw. Wirkung von online verbreiteten Inhalten der Gesetzgeber die zumindest nicht irrelevante Verfügbarkeit von Inhalten in der Öffentlichkeit als Basis für Regulierung nehmen.

62 Vgl. beispielsweise die Definition entwicklungsbeeinträchtigender Angebote in $\S 5 \mathrm{JMStV}$, die nur „geeignet“ sein müssen, diesen Effekt zu haben, wobei eine solche Eignung „,vermutet“ (Abs. 2) wird, wenn sie keine Freigabe für die jeweilige Altersstufe haben. Hartstein u.a., in: HK-RStV, § 5 JMStV Rn. 12. 